\title{
Elevated Ratios of Type I/III Collagen in the Lungs of ChronicallyVentilated Neonates with Respiratory Distress
}

\author{
CRAIG T. SHOEMAKER, KAREN M. REISER, BOYD W. GOETZMAN, AND JEROLD A. LAST \\ Departments of Pediatrics and Medicine, and California Primate Research Center, University of California,
} Davis, California 95616

\begin{abstract}
Increased synthesis of type I collagen, leading to increased ratios of type I to type III collagen in the lungs, has been observed in the lungs of animals with experimental pulmonary fibrosis. Similar changes in collagen type ratios have been observed in lungs of humans dying of idiopathic pulmonary fibrosis and of adult respiratory distress syndrome. In this study, lung collagen type ratios were examined in infants with acute and chronic lung disease. Tissue from the right lower lobes of neonates was obtained post mortem. Specific collagen types were quantitated by solubilization of lung collagen with $\mathrm{CNBr}$ and fractionation of the resulting mixture of peptides by column chromatography and polyacrylamide gel electrophoresis. Ratios of type I/III collagen were calculated for each lung sample using two independent pairs of marker peptides for these determinations. In some cases the ratio of type $V$ to type III collagen in these same lung samples was also quantitated. We observed a significant increase in the ratio of type $\mathrm{I} / \mathrm{III}$ collagen in infants with a premortem diagnosis of chronic lung disease, usually preceded by respiratory distress syndrome. We also observed two infants with large changes in collagen type ratios who might have had pulmonary fibroplasia secondary to intrauterine lung disease. These data suggest that there may be several subsets of infants with respiratory distress syndrome, each having a different prognosis. (Pediatr Res 18:1176-1180, 1984)
\end{abstract}

\begin{abstract}
Abbreviations
RDS, respiratory distress syndrome

ARDS, adult respiratory distress syndrome

CLD, chronic lung disease

TPN, total parenteral nutrition
\end{abstract}

Respiratory distress syndrome (hyaline membrane disease) is the leading cause of morbidity and mortality in prematurely born infants. Dramatic advances in our understanding of the pathophysiological basis of this disease (and its successful treatment in neonatal intensive care units) have led to a tremendous improvement in the prognosis for RDS. One such treatment has been

Received February 13, 1984; accepted April 24, 1984.

Correspondence may be addressed to Dr. Jerold A. Last, California Primate Research Center, University of California, Davis, CA 95616.

This study was supported in part by Grants ES-00628 and RR-00169 from the National Institutes of Health. K.M.R. was supported by Young Investigator Award HL-26795 from the National Heart, Lung, and Blood Institute. prenatal administration of corticosteroids (1). Infants afflicted with RDS often require mechanical ventilation with positive pressure and high partial pressures of oxygen to maintain respiratory exchange. Northway and his colleagues (2) described the complications associated with the therapy of RDS, calling the resulting syndrome bronchopulmonary dysplasia. A prominent pahological feature of this syndrome is the presence of thickened alveolar walls containing excess collagen fibers, i.e. interstitial pulmonary fibrosis. Northway et al. (2) recognized the possibility that bronchopulmonary dysplasia might be an iatrogenic disease secondary to oxygen toxicity. At the same time, Nash et al. (3) described pulmonary fibrosis associated with treatment of ARDS with oxygen therapy and mechanical ventilation.

Our interest in collagen types in lungs of infants suffering from RDS stems from several observations in our laboratory. In different animal models of experimentally induced acute respiratory disease, we have observed shifts in the types of collagens being synthesized in lungs of affected animals, in conjunction with increased amounts of collagen (4). We have recently shown (5) that lungs from patients with ARDS also contain increased ratios of type I to type III collagen as compared with controls. Seyer et al. (6) have previously reported that similar shifts of collagen type ratios occur in lungs from adult patients dying of idiopathic pulmonary fibrosis. In addition, Zapol and coworkers (7) have reported that lungs of patients with ARDS contain larger amounts of collagen than lungs from normal subjects.

In the present study, we report upon the ratio of collagen types in lungs from newborn infants dying with evidence of pulmonary fibrosis (seven of nine also having had previous RDS) as compared with the ratios in lungs from infants dying of other causes without pulmonary fibrosis. New methods for collagen typing are used to quantitate the relative amount of type $\mathrm{V}$ collagen present in some of these lungs, and to evaluate the ratio of type I to type III collagen independently of our previously used standard methods.

\section{MATERIALS AND METHODS}

Right lower lobes or pieces thereof of lungs from 19 human infants, three of whom were stillborn, were dissected out during routine autopsy examination and placed in a sealed polyvinylchloride container, frozen, and stored at $-18^{\circ} \mathrm{C}$. Specimens were later thawed, weighed, and subjected to $\mathrm{CNBr}$ digestion, ionexchange chromatography on carboxymethyl cellulose, and polyacrylamide gel electrophoretic analysis according to the methods of Reiser and Last $(4,8,9)$. Using as index peptides $\alpha_{1}(\mathrm{I}) \mathrm{CB}-8$ $3, \alpha_{1}(\mathrm{I}) \mathrm{CB}-8, \alpha_{1}(\mathrm{III}) \mathrm{CB}-8$, and $\alpha_{1}(\mathrm{~V}) \mathrm{CB}-\mathrm{a}$, we were able to quantitate molar ratios of types I, III, and V collagen in the tissue samples. In addition, we independently evaluated ratios of type I to type III collagen in the same tissue samples using as index 
peptides $\alpha_{1}$ (I)CB-7, $\alpha_{1}$ (I)CB-3-7, and $\alpha_{1}$ (III)-CB-5 (10). Since tissue samples were usually not entire lobes, we did not attempt to quantitate collagen (hydroxyproline) content of the lung lobes. Cyanogen bromide quantitatively solubilized $(>95 \%)$ the hydroxyproline from infant lung lobes under the conditions (1.5:1 $\mathrm{CNBr}$ :dry weight of sample lung; $4 \mathrm{~h}$ of digestion at room temperature) used in this study.

Patients in this study had various neonatal (and prenatal) diseases. This protocol was approved by the University Committee on Ethics of Human Experimentation and informed consent was obtained from parents for acquisition of tissue for this study. Clinical and pathological diagnoses potentially relevant to lung collagen content and to cause(s) of death are listed for all of the subjects in this study in Table 1. Group 1 died of causes other than pulmonary fibroplasia (chronic lung disease) and comprised 10 infants with a mean birth weight of $1899 \pm 959 \mathrm{~g}$ and a mean gestational age of $33.7 \pm 7.6 \mathrm{wk}$. Group 2 had clinically or pathologically defined pulmonary fibroplasia (11-13), and included nine infants with a mean birth weight of $1232 \pm 863 \mathrm{~g}$ and a mean gestational age of $29.0 \pm 3.8 \mathrm{wk}$. All analyses were performed on coded samples without knowledge of the diagnosis for each infant. The results were subsequently correlated with clinical and histopathological information obtained from chart review by an individual other than the one performing the biochemical assays.

We examined several subsets of patients despite the relatively small numbers involved to seek correlations other than presence of infant RDS with changes in collagen type I/III ratios. Medical records were reviewed to determine maternal gestational history including gestational complications and medication use, infant birth weight, gestational age, total ventilator days, diagnoses during life, medications utilized, radiographic, and histologic findings. Ventilator pressures and inspired percentage of oxygen were not analyzed due to the complex alterations that occurred with each patient.

\section{RESULTS}

We determined ratios of types I and III collagen from lungs of all infants in this study (Table 2). For the control infants $(n=$ $10)$, the group mean $( \pm S D)$ was $1.62 \pm 0.14: 1.0$ for the observed ratio of type I/type III collagen. This value may be compared

Table 1. Clinical history of patients in study

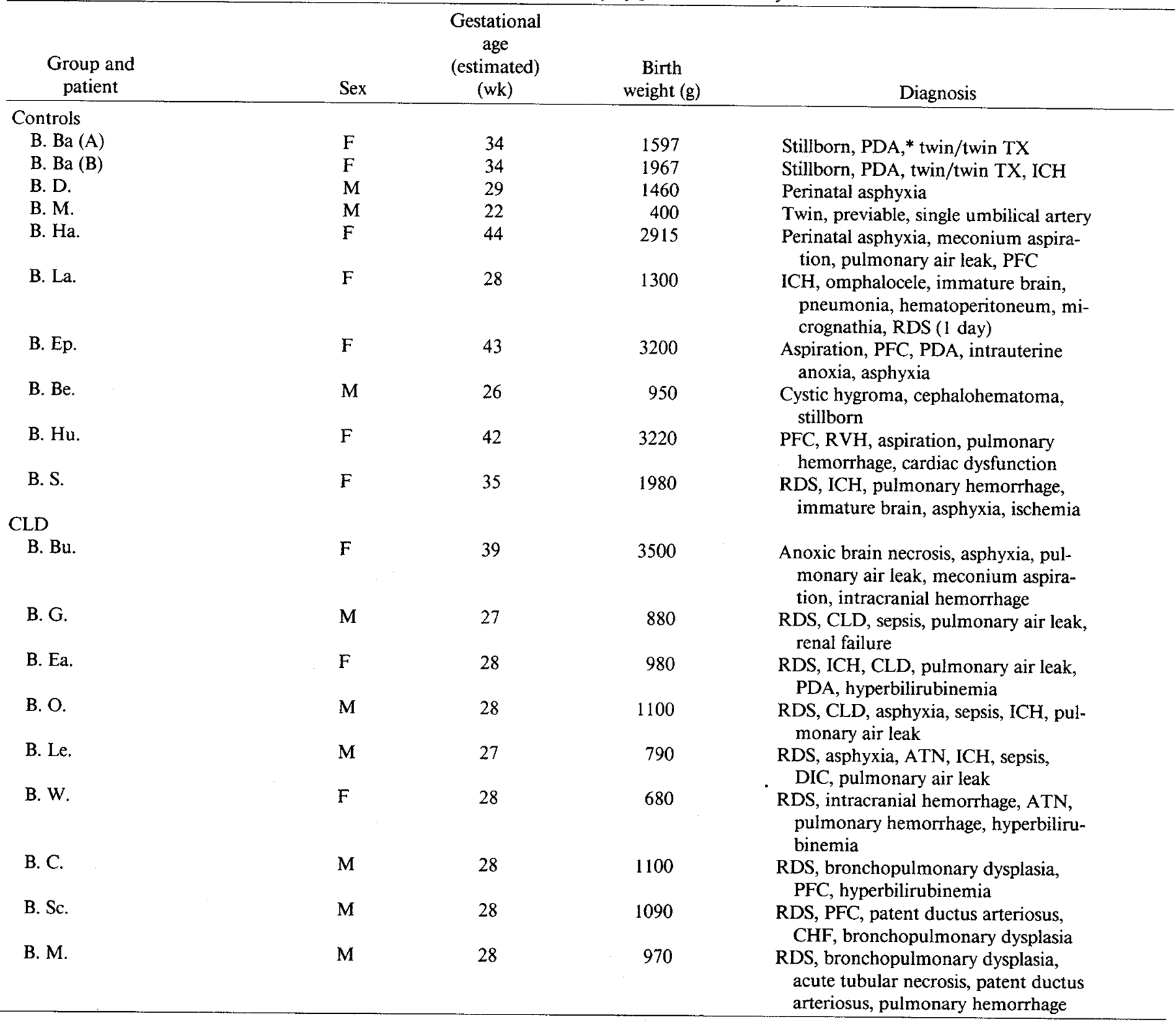

*PDA, patent ductus arteriosus; TX, transfusion; ICH, intracranial hemorrhage; PFC, persistence of fetal circulation; RVH, right ventricular hypertrophy; DIC, disseminated intravascular coagulation; ATN, acute tubular necrosis; CHF, congestive heart failure 
Table 2. Collagen type ratios in lungs from neonates with CLD*

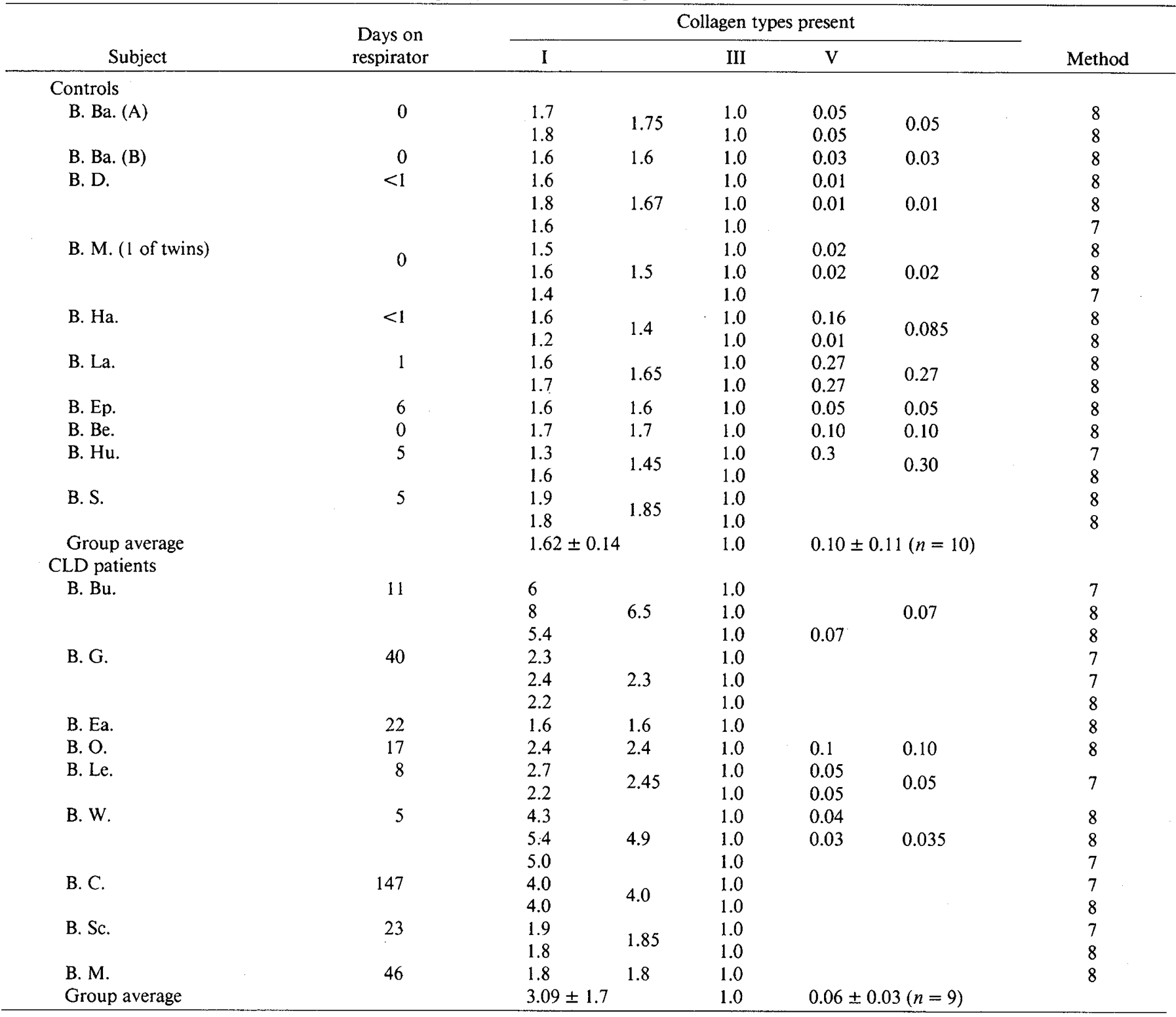

* Method: 7, CB-7, 3-7:CB-5 ratios; 8, CB-8, 3-8:CB-8 ratios (see text for details).

with the normal value of $2.00 \pm 0.14: 1.0$ that we have previously observed (5) for adult human lungs $(n=8)$ using these same techniques, and is consistent with the relatively higher content of type III collagen expected in fetal and/or neonatal lung tissue than in adult (14). For the infants with CLD $(n=9)$, we found the comparable ratio of collagen types to be $3.09 \pm 1.7: 1$, significantly (Student's $t$ test; $p<0.01$ ) higher than the value for control lungs. It is noteworthy that except for a single value (Baby Ea.), all CLD infant lungs had higher ratios of type I/type III collagen than any of the control lungs. The observed values were also consistent for two independent sets of marker peptides: the $\alpha_{1}$ (III)CB- $8, \alpha_{1}$ (I)CB-8, $\alpha_{1}$ (I)CB-8-3 set we have used previously, and the $\alpha_{1}$ (III)CB-5, $\alpha_{1}$ (I)CB-7 set we also used in the present study. Due to their similar molecular weights, the CB-7, CB-5 pair offers theoretical and practical advantages as compared with the CB-8, CB-8 pair (10).

We also examined ratios of collagens type V/type III in lung samples where enough tissue was available to make this assay practical. We found a value of $0.10 \pm 0.11: 1.0(n=9)$ for this ratio in control lungs, a value that may be compared with our previous observation (9) of $0.14 \pm 0.02: 1.0(n=4)$ in rat lungs.
For infants with CLD, the comparable ratio was 0.06 $\pm 0.03: 1.0$ $(n=4)$, not significantly different from the control value.

Within the subset of ventilated patients, single parameters were selected to compare the collagen ratios of that group to the entire subset of non-CLD patients. Parameters selected included birth weights less than $1000 \mathrm{~g}$, diagnoses of chronic lung disease, evidence of pulmonary air leak, persistent fetal circulation, bacterial sepsis during ventilation, congenital bacterial pneumonia, central hyperalimentation, perinatal asphyxia, and intracranial hemorrhage. Means of the ratios of collagen types I/III for each group were then compared using a one-tailed $t$ test with a pooled variance technique for nonmatched samples. Means were obtained by averaging all specimens obtained from a single patient and using the mean value for each patient to determine the collective mean. The results of such data analysis are shown in Table 3. Significant association of changes in collagen type ratio were found with two subsets of the data: babies requiring total parenteral nutrition $(n=5)$ and babies with documented sepsis $(n=5)$.

In a further effort to examine patient subsets, we prepared scattergrams of data relating collagen type ratios to conceptual 
Table 3. Comparison of means of identified subsets to no identified pulmonary fibrosis

\begin{tabular}{|c|c|c|}
\hline Diagnosis subset $(n)$ & $\begin{array}{c}\text { Mean Ratio I/ } \\
\text { III } \\
( \pm \mathrm{SD})\end{array}$ & $p$ value* \\
\hline Not ventilated (3) & $1.62 \pm 0.12$ & NS \\
\hline $\begin{array}{l}\text { Ventilated less than } 8 \text { days } \\
\text { (9) }\end{array}$ & $1.91 \pm 0.99$ & NS \\
\hline $\begin{array}{l}\text { Ventilated more than } 7 \\
\text { days (8) }\end{array}$ & $2.87 \pm 1.63$ & NS \\
\hline CLD (9) & $3.08 \pm 1.67$ & NS \\
\hline Asphyxia (8) & $2.46 \pm 1.66$ & NS \\
\hline TPN (5) & $2.47 \pm 0.89$ & $<0.05$ \\
\hline Birth weight $<1000 \mathrm{~g}(7)$ & $2.32 \pm 1.92$ & NS \\
\hline $\operatorname{RDS}(9)$ & $2.31 \pm 1.02$ & NS \\
\hline Pulmonary air leak (10) & $2.59 \pm 1.55$ & NS \\
\hline Sepsis (5) & $3.21 \pm 1.17$ & $<0.001$ \\
\hline $\mathrm{ICH}(7)$ & $2.36 \pm 1.17$ & NS \\
\hline Steroids (4) & $2.51 \pm 1.03$ & $<0.025$ \\
\hline PFC (9) & $2.56 \pm 1.67$ & NS \\
\hline Congenital pneumonia (3) & $1.54 \pm 0.12$ & NS \\
\hline
\end{tabular}

* By a one-tailed $t$ test, with pooled variance. ICH, intracranial hemorrhage; PFC, persistence of fetal circulation.

age at death and to total days of mechanical ventilation, as shown in Figure 1. Two subjects were observed that had markedly increased collagen type I/III ratios, only one of whom had clinical evidence of chronic lung disease. These individuals will be discussed below in more detail.

\section{DISCUSSION}

Up to $85 \%$ of the connective tissue mass of lung is collagen, of which type I and type III collagen are the predominant types (14). Type I collagen, histologically defined "collagen" that stains with Masson's trichrome, is less compliant than type III collagen, which is thought to be part of the complex that stains as reticulin (15). The lungs of infants with pulmonary fibroplasia contain relatively more type I than type III collagen as compared with lungs of infants dying of other causes (Table 2). By analogy with adults dying of ARDS $(5,7)$, their lungs also presumably contain more collagen, and it is this excess type I collagen that accounts for the change in ratios of collagen types present (4). This excess of type I collagen accounts for the histologically apparent pulmonary fibrosis that is a hallmark of chronic lung disease in infants that cannot be rapidly weaned from ventilator support.

There are several potential difficulties in interpretation of the data from a clinical study such as this one. First, is the control group truly an appropriate one? It is older ( $34 \pm 8$ versus $29 \pm 4$ weeks of estimated gestational age) and larger ( $1.9 \pm 1$ versus 1.2 $\pm 0.9 \mathrm{~kg}$ ) than the group composed of infants with CLD. Given that RDS is a disease of prematurity, there is little that one can do about this disparity except to note that the younger and smaller infants in the control group (Babies $\mathrm{M}$. and Be.) had values for collagen type ratios that were typical of the group as a whole. In addition, the effect of a younger gestational age should be to decrease the type I/III ratio, as there is relatively more type III collagen in fetal tissue than in adult (14). Thus, any error introduced by having an older control group than CLD group should be to underestimate the true difference in collagen type ratios in their lungs. In addition, we did not observe any trend when correlating collagen type ratios and conceptual age of subjects (Fig. 1A). Therefore, we can conclude that the control group was not only appropriate, but a conservative choice.

Second, is the chosen methodology appropriate? We have discussed elsewhere the need for $\mathrm{CNBr}$ peptide-mapping techniques to quantitate lung collagen types due to the extreme insolubility of lung collagen $(4,8,9)$, and have documented the validity of the methods used in the present study for tissue from
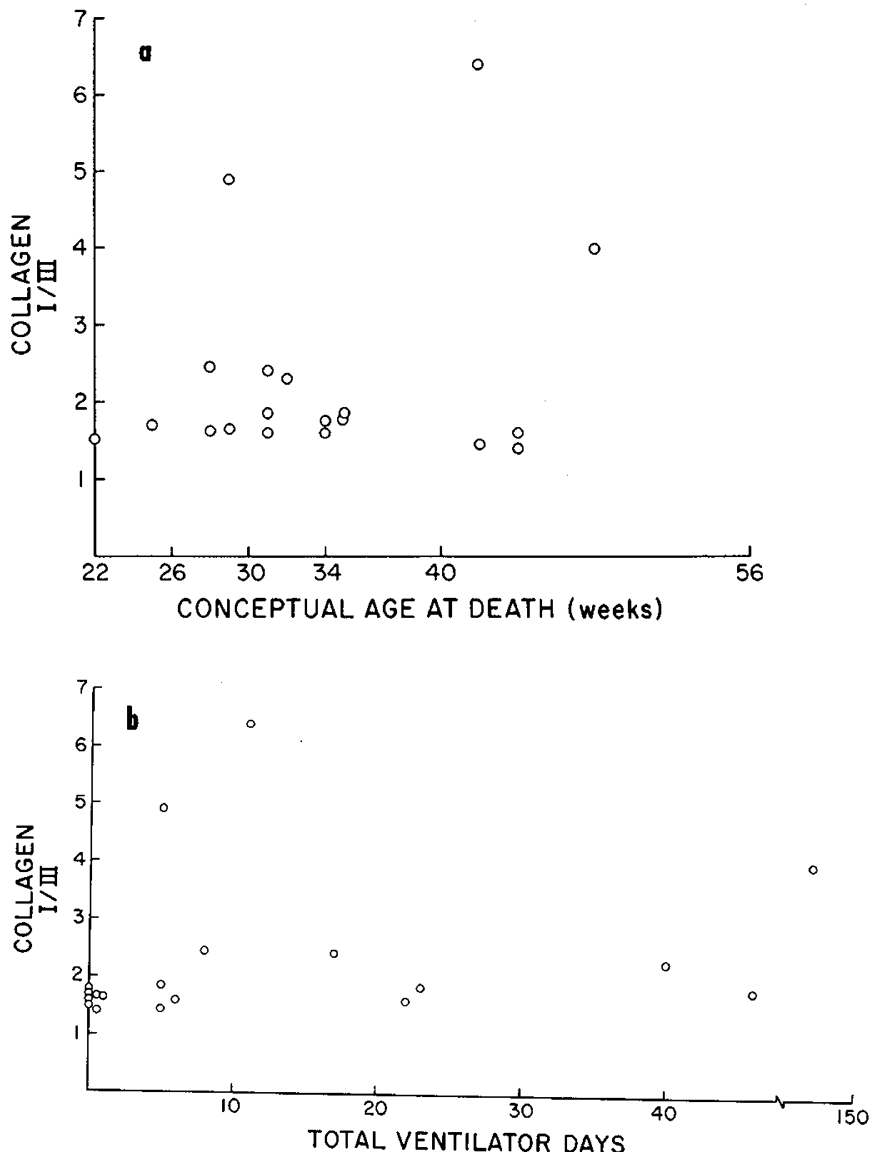

Fig. 1. Lung collagen type ratios as a function of $(A)$ conceptual age at death or $(B)$ total ventilator days (of treatment) for the 19 patients studied. Babies specifically discussed in the text include three with ratios of $4: 1$ or greater as shown: these were Baby W., 28 wk and 5 ventilator days; Baby Bu., 41 wk and 11 ventilator days; and Baby C., 49 wk and 147 ventilator days.

both laboratory rodents and human lungs $(4,5)$. We have confirmed these results in the present study by introducing an independent evaluation of collagen type I/III ratios, analysis of $\alpha_{1}(\mathrm{I})$ CB-7, $\alpha_{1}(\mathrm{I})$-CB-3-7: $\alpha_{1}$ (III)CB-5 ratios. As shown in Table 2, these values are identical to those found with our previously used marker peptides, $\alpha_{1}(\mathrm{I}) \mathrm{CB}-8, \alpha_{1}(\mathrm{I}) \mathrm{CB}-8-3$, and $\alpha_{1}(\mathrm{III}) \mathrm{CB}-8$.

In our analysis of subgroups of infants in this study, we observed three lung samples with extremely high collagen type I/III ratios (>4-5; $c f$. Fig. 1, $A$ and $B$ ). One infant's high collagen type ratio is consistent with a clinical history of RDS, bronchopulmonary dysplasia, and 147 days of ventilator support with a high percentage of inspired $\mathrm{O}_{2}$. The other two infants, however, seem to have anomalously high values for their collagen type ratios. How can we explain values greater than 5:1 for these two infants, one "without clinically apparent chronic lung disease"? Based upon detailed review of their charts and histopathological review of autopsy tissue, we suggest that these two babies had "intrauterine shock lung." Both babies had evidence of asphyxia and CNS damage in utero. In the case of one of these infants (Baby Bu.), we can estimate occurrence of the intrauterine event from an amniocentesis performed 2 wk prior to the delivery date, as there were changes in the fetal activity stemming from that time. The pathological findings for this infant included CNS glial proliferation and evidence of fetal/maternal transfusion with fetal anemia, both conditions associated with prolonged in utero hypoxemia (19).

Type V/III collagen ratios in control lungs did not differ significantly from ratios in lungs from neonates with CLD (Table 2 ). However, the relatively small amount of type V collagen in the lung (less than $2-3 \%$ of the total lung collagen) and the low 
molar yield (about $10 \%$ ) of the marker peptide $\alpha_{1}(\mathrm{~V}) \mathrm{CB}-\mathrm{a}(9)$ make this a very insensitive assay for small changes in type $\mathrm{V}$ collagen content of the lung. Clearly, the peptide mapping methods for type $\mathrm{V}$ collagen must be refined to increase the signal-tonoise ratio in this assay before rigorous conclusions about relative ratios of this minor collagen type can be drawn. Qualitative changes in type $\mathrm{V}$ collagen in adult fibrotic lungs have been reported in immunofluorescence studies that have used anticollagen antibodies (16).

The highly significant association of changes of collagen type ratios (Table 3) with TPN was probably factitious. The TPN sample was probably subject to a strong selection bias in that a chronically ill infant would be started on this therapeutic modality. We observed an inverse association with the use of corticosteroids, but in three of these four cases steroid treatment was used as an adjunctive therapy late in the course of the disease. An interesting and unexpected finding was a strong correlation between bacterial infection during mechanical ventilation and subsequent CLD. Congenital pneumonia was unrelated to CLD in this study. We may speculate that the infant with RDS and damaged pulmonary endothelium is a subject at high risk for bacterial proliferation in the lung, perhaps due to compromised host lung defenses accompanying this disease.

The results of Figure $1 B$ seem to rule out any direct causal association between duration of mechanical ventilation and elevated ratios of type I to type III collagen in the lungs of the RDS group. It has not been possible to analyze $\mathrm{O}_{2}$ administration data in the same fashion due to the complex patterns of its usage, but we feel that it too can be ruled out as a direct causal factor based on its close correlation with ventilator usage (days) in most cases and our previous results in adults dying of diseases other than ARDS who required $\mathrm{O}_{2}$ therapy and ventilator assistance (5).

In conclusion, we have identified at least two groups of infants dying of CLD, one in which RDS and alterations of collagen synthesis apparently lead to pulmonary fibrosis, and a second group in which very high levels of type I collagen synthesis may have occurred in utero after prolonged fetal hypoxemia. It is therefore possible that increased lung collagen content in CLD after RDS in neonates, as well as ARDS, is not due to oxygen therapy and mechanical ventilation with barotrauma, but to the underlying disease itself. It is possible that in neonates at high risk for CLD pharmacological intervention at the time of initial injury with drugs that alter collagen synthesis or deposition might be appropriate therapy for the prevention of lung fibroplasia. Steroids have been shown to decrease collagen synthesis is damaged animal lung (17) and, in some studies, to improve pulmo- nary function in human infants with bronchopulmonary dysplasia (18). The possibility that corticosteroids might be effective in decreasing the rate of collagen synthesis in the lung if utilized prior to the injuring stimulus or during the phase of acute injury may warrant a clinical trial in neonates that are at high risk for pulmonary fibroplasia.

\section{REFERENCES}

1. Ballard PL, Granberg P, Ballard RA 1975 Glucocorticoid levels in maternal and cord serum after prenatal betamethasone therapy to prevent respiratory distress syndrome. J Clin Invest 56:1548

2. Northway WH, Jr, Rosan RC, Porter DY 1967 Pulmonary disease following respiratory therapy of hyaline-membrane disease: bronchopulmonary dysplasia. N Engl J Med 276:357

3. Nash G, Blennerhassett JB, Pontoppidan H 1975 Pulmonary lesions associated with oxygen therapy and artificial ventilation. N Engl J Med 276:368

4. Reiser KM, Last JA 1981 Pulmonary fibrosis in experimental acute respiratory disease. Am Rev Respir Dis 123:58

5. Last JA, Siefkin AD, Reiser KM 1983 Type I collagen content is increased in lungs of patients with adult respiratory distress syndrome. Thorax 38:364

6. Seyer JM, Hutcheson ET, Kang AH 1976 Collagen polymorphism in idiopathic chronic pulmonary fibrosis. J Clin Invest 57:1498

7. Zapol WM, Trelsted RL, Coffey JW, Tsai L, Salvador RA 1979 Pulmonary fibrosis in severe acute respiratory failure. Am Rev Respir Dis 119:547

8. Reiser KM, Last JA 1980 Quantitation of specific collagen types from lungs of small mammals. Anal Biochem 104:87

9. Reiser KM, Last JA 1983 Type V collagen. Quantitation in normal lungs and in lungs of rats with bleomycin-induced pulmonary fibrosis. J Biol Chem 258:269

10. Hanson AN, Bentley JP 1983 Quantitation of type I to type III collagen ratios in small samples of human tendon, blood vessels, and atherosclerotic plaque. Anal Biochem 130:32

11. Bannerjee CK, Girling DJ, Wigglesworth JS 1972 Pulmonary fibroplasia in newborn babies treated with oxygen and artificial ventilation. Arch Dis Child 47:509

12. Edwards DK, Colby TV, Northway WH 1979 Radiologic-pathologic correlation in bronchopulmonary dysplasia. J Pediatr 95:834

13. Bonikos DS, Bensch KG, Northway WH Jr, Edwards DK 1976 Bronchopulmonary dysplasia: the pulmonary pathological sequel of necrotizing bronchiolitis and pulmonary fibrosis. Hum Pathol 7:643

14. Hance A, Crystal RG 1975 The connective tissue of lung. Am Rev Respir Dis 112:657

15. Greenberg DB, Reiser KM, Last JA 1978 Correlation of biochemical and morphologic manifestations of acute pulmonary fibrosis in rats administered paraquat. Chest $74: 421$

16. Madri JA, Furthmayr H 1980 Isolation and tissue localization of type $A B$ collagen from normal lung parenchyma. Hum Pathol 11:353

17. Hesterbert TW, Last JA 1981 Ozone-induced acute pulmonary fibrosis in rats. Prevention of increased rates of collagen synthesis by methylprednisolone Am Rev Respir Dis 123:47

18. Sobel DB, Lewis K, Deming DD, McCann EM 1983 Dexamethasone improves lung function in infants with chronic lung disease. Pediatr Res 17:390A (abstr)

19. Friede R 1975 Developmental Neuropathology. Springer-Verlag, Heidelberg, pp $51-75$ 